\title{
Interações medicamentosas em prescrições de idosos hipertensos: prevalência e significância clínica
}

\section{Drug interactions in prescriptions for elderly hypertensive patients: prevalence and clinical significance}

\section{Interacciones medicamentosas en prescripciones de ancianos hipertensos: prevalencia y significado clínico}

\author{
Natália Balera Ferreira Pinto'; Liliana Batista Vieirall; Fernanda Maria Vieira Pereira \\ Adriano Max Moreira Reis ${ }^{I V}$; Silvia Helena De Bortoli Cassiani ${ }^{V}$
}

\begin{abstract}
RESUMO: O objetivo deste estudo foi analisar a prevalência e significância clínica de interações medicamentosas identificadas nas prescrições de pacientes idosos hipertensos. Trata-se de um estudo descritivo, realizado na Unidade Básica de Saúde Dom Mielle da cidade de Ribeirão Preto, Estado de São Paulo, de julho a dezembro de 2011. As interações foram identificadas empregando o software Drug-Reax® da Micromedex e classificadas quanto à gravidade, documentação e tempo de início. Utilizou-se o software SAS®9.0 para análise estatística descritiva. Dos 40 pacientes do estudo, 65\% eram mulheres e a mediana de idade foi 71 anos ( $D P=5,9)$. A média de medicamentos prescritos por paciente foi de $7,5(\mathrm{DP}=2,8)$. Todos apresentaram, no mínimo, uma interação medicamentosa. Do total de 169 interações identificadas, 17,1\% eram graves. A ocorrência de interações medicamentosas foi elevada nos pacientes investigados. A equipe de saúde deve identificar as interações de significância clínica e implementar medidas para a sua prevenção e monitorização.

Palavras-Chave: Interações de medicamentos; idoso; hipertensão; atenção primária à saúde.
\end{abstract}

ABSTRACT: This study examined the prevalence and clinical significance of drug interactions identified in prescriptions of elderly hypertensive patients. This descriptive study was conducted at the Dom Mielle Primary Health Care Unit in Ribeirão Preto, São Paulo State, from July to December 2011. Interactions were identified using Drug-Reax® Micromedex software and classified by severity, time of onset and documentation. SAS $₫ 9.0$ software was used for descriptive statistics. Of the 40 patients studied, $65.0 \%$ were women and the median age was $71.0(\mathrm{SD}=5.9)$ years. The average number of drugs prescribed per patient was $7.5(\mathrm{SD}=2.8)$; all patients had at least one drug interaction. Of the 169 interactions identified, $17.1 \%$ were severe. The occurrence of drug interactions was high in the patients studied. Healthcare teams must identify drug interactions of clinical significance and implement measures to prevent and monitor them.

Keywords: Drug interactions; older adults; hypertension; primary health care.

RESUMEN: El objetivo del estudio fue analizar la prevalencia y relevancia clínica de interacciones de drogas identificadas en las prescripciones de pacientes ancianos con hipertensión. Se trata de un estudio descriptivo, realizado en la Unidad Básica de Salud Dom Mielle en la ciudad de Ribeirão Preto, Estado de São Paulo - Brasil, de julio a diciembre de 2011. Las interacciones se identificaron usando el software Drug-Reax® Micromedex y clasificados por la gravedad, el tiempo de inicio y la documentación. Se utilizó el software SAS®9.0 para estadístico descriptivo. De los 40 pacientes estudiados, 65\% eran mujeres y la edad mediana fue de $71,0(\mathrm{SD}=5,9)$ años. El número promedio de medicamentos prescritos por paciente fue de 7,5 (SD=2,8). Todos tenían, al menos, una interacción medicamentosa. Del total de 169 interacciones, 17,1\% fueron graves. La aparición de las interacciones de drogas fue alta en los pacientes investigados. El equipo de salud debe identificar las interacciones farmacológicas de importancia clínica y aplicar medidas para su prevención y control.

Palabras Clave: Interacciones de drogas; anciano; hipertensión; atención primaria a la salud.

\section{INTRODUÇÃO}

Com o avançar da idade, as pessoas acabam desenvolvendo doenças crônicas como hipertensão, diabetes, hipercolesterolemia e doenças cardiovasculares e acabam utilizando uma grande quantidade de medicamentos, os quais podem trazer benefícios, quando usados corretamente pelo paciente, e podem trazer prejuízos se não utilizados adequadamente.

${ }^{\mathrm{I} G r a d u a n d a ~ d a ~ E s c o l a ~ d e ~ E n f e r m a g e m ~ d e ~ R i b e i r a ̃ o ~ P r e t o ~ d a ~ U n i v e r s i d a d e ~ d e ~ S a ̃ o ~ P a u l o . ~ R i b e i r a ̃ o ~ P r e t o, ~ S a ̃ o ~ P a u l o, ~ B r a s i l . ~ E-m a i l: ~ n a t a l i a b f p @ y a h o o . c o m . b r . ~}$ IIFarmacêutica, Doutora em Ciências pela Escola de Enfermagem de Ribeirão Preto da Universidade de São Paulo. Ribeirão Preto, São Paulo, Brasil. E-mail: lilianabvieira@yahoo.com.br.

IIIEnfermeira, Doutoranda em Enfermagem do Programa Interunidades de Doutoramento em Enfermagem da Escola de Enfermagem de Ribeirão Preto da Universidade de São Paulo. Ribeirão Preto, São Paulo, Brasil. E-mail: fernanddamaria@hotmail.com.

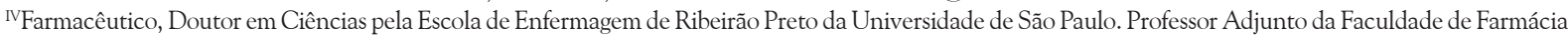
da Universidade Federal de Minas Gerais. Departamento de Produtos Farmacêuticos. Belo Horizonte, Minas Gerais, Brasil. E-mail: amreis@outlook.com. vEnfermeira, Professora Doutora da Escola de Enfermagem de Ribeirão Preto. Regional Advisor on Nursing and Allied Health Personnel, Pan American Health Organization. São Paulo, Brasil. E-mail: sbhcassi@eerp.usp.br.

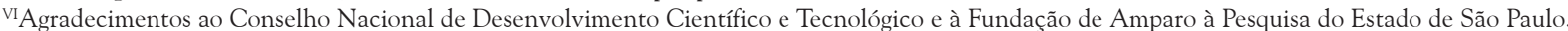


Esse estudo fez parte de um projeto de doutorado desenvolvido pela Escola de Enfermagem de Ribeirão Preto, da Universidade de São Paulo, o qual avaliou a adesão à terapêutica medicamentosa após uma intervenção na dispensação dos medicamentos. Durante o processo de acompanhamento farmacoterapêutico dos pacientes desse projeto, sentiu-se a necessidade de estudar as interações entre os medicamentos utilizados por esses participantes a fim de garantir o uso racional.

O uso de vários tipos de medicamentos pode provocar interações entre eles, as quais podem melhorar ou prejudicar a ação farmacológica, trazendo consequências para o paciente. Para evitar consequências graves é importante que os profissionais de saúde como médicos, farmacêuticos e enfermeiros conheçam os tipos de interações que podem ocorrer entre os medicamentos disponibilizados nas unidades de atenção primária à saúde e os processos de monitorização para que possam garantir a segurança da terapia medicamentosa e assim, a segurança do paciente.

Nesse contexto, com o intuito de contribuir para o conhecimento sobre o assunto, o presente estudo teve como objetivo - a partir das prescrições médicas de pacientes idosos hipertensos, atendidos em uma unidade básica de saúde (UBS) do interior do Estado de São Paulo, analisar a prevalência de potenciais interações medicamentosas do tipo fármaco-fármaco e sua significância clínica.

\section{REVISÃo DE LITERATURA}

A proporção de idosos vem aumentando na população do Brasil, e este envelhecimento tem como explicação a continuação do processo de declínio da fecundidade e simultaneamente, o crescimento da expectativa de vida, tanto dos homens como das mulheres ${ }^{1}$. A população idosa, devido às alterações cognitivas e físicas associadas ao envelhecimento, tem um risco aumentado para problemas de aderência à medicação e pode apresentar dificuldades no uso de medicamentos. A polifarmácia e a inadequação da terapêutica, além de aumentarem a demanda dos serviços de saúde e aumentarem os riscos de reações adversas e interações medicamentosas, favorecem a internação hospitalar ou contribuem para ampliar a sua duração ${ }^{2}$, elevando os custos da saúde ${ }^{3-5}$.

O aumento da população idosa colabora para a alta prevalência de inúmeras e variadas doenças cujos tratamentos, na maioria das vezes, incluem recursos farmacológicos ${ }^{4}$. Entre as várias doenças crônicas que os idosos apresentam destaca-se a hipertensão arterial, que também requer custos médicos e socioeconômicos elevados, decorrentes, principalmente, das suas complicações, tais como: doença cerebrovascular, doença arterial coronariana, insuficiência cardíaca, insuficiência renal crônica e doença vascular de extremidades ${ }^{6}$.
A gravidade, prevalência e possíveis consequências das interações medicamentosas estão relacionadas a variáveis como condições clínicas dos indivíduos e número e características de medicamentos ${ }^{7,8}$. A população idosa é alvo de significativa prevalência das interações que demandam a avaliação da possibilidade de uso de alternativas terapêuticas, farmacológicas ou não, ou de alterações nas doses ou na via de administração dos medicamentos envolvidos 4 .

Dessa forma, torna-se importante identificar as potenciais interações no tratamento da hipertensão arterial e outras doenças crônicas e realizar manejo farmacoterapêutico adequado para evitar efeitos adversos graves. Os profissionais de saúde precisam estar atentos às interações entre fármacos e precisam estar aptos a descrever o resultado das potenciais interações e sugerir apropriadas intervenções ${ }^{8,9}$.

\section{Metodologia}

Estudo descritivo, observacional e transversal realizado na Unidade Básica de Saúde Dom Mielle da cidade de Ribeirão Preto, no estado de São Paulo, de julho a dezembro de 2011. A partir do banco de dados de informações de saúde do sistema informatizado do município, foi gerada uma planilha no programa Excel ${ }^{\circledR}$ contendo a lista dos pacientes idosos, com 60 anos ou mais.

Os critérios para inclusão no estudo foram: pacientes com 60 anos ou mais; uso contínuo de quatro ou mais medicamentos diferentes, sendo pelo menos um anti-hipertensivo; hipertensos com pressão arterial sistólica (PAS) $\geq 130 \mathrm{mmHg}$; usuários dos serviços da farmácia da UBS e em acompanhamento médico. Foram excluídos os pacientes usuários de insulina e aqueles incapazes de administrar seus próprios medicamentos, dependentes de outras pessoas para fazê-lo. $\mathrm{O}$ critério de inclusão de quatro ou mais medicamentos visou garantir a participação de idosos com polifarmacoterapia moderada ou alta, adotando os pontos de corte - moderada de 4 a 5 e alta superior a $5^{10,11}$.

A partir da lista do programa Excel $\AA$, foram pré-selecionados 265 pacientes. Após tentativas de contato telefônico, 28 não quiseram participar; 19 apresentavam dificuldade em andar; 10 mudaram de área; um morava no asilo; dois apresentavam problemas mentais; nove trabalhavam o dia todo e não poderiam participar; quatro faleceram; 34 não compareceram ao primeiro encontro (foram feitas três tentativas); 53 não foram encontrados e 105 pacientes compareceram ao primeiro encontro e foram atendidos na UBS. Nesse atendimento, realizou-se a aferição da pressão arterial por três vezes, excluindo a primeira medida e calculando a média das duas últimas. Foi usado um aparelho digital calibrado e validado, modelo Omron HEM-742. Desses 105 pacientes, 59 apresen- 
tavam PAS $<130 \mathrm{mmHg}$, seis não quiseram participar e 40 preencheram os critérios de inclusão. Assim, a população do estudo foi composta por 40 pacientes.

Foram realizados dois encontros com cada paciente: o primeiro na pré-seleção e o segundo para coleta dos dados. As entrevistas foram feitas na UBS, em uma sala reservada, e com uma duração média de 30 minutos. Foi aplicado um instrumento composto por informações sociodemográficas; o tempo de diagnóstico da hipertensão e as comorbidades apresentadas também foram transcritas todas as prescrições do paciente em um instrumento contendo a data da prescrição, o número da identificação do paciente, os nomes dos medicamentos, a dose, a frequência, o horário e o tempo de uso. Foi realizado um estudo piloto com o intuito de testar os instrumentos de coleta de dados com cinco pacientes da UBS, selecionados aleatoriamente.

As interações medicamentosas, do tipo fármacofármaco, presentes nas prescrições dos idosos, foram identificadas empregando o software Drug-Reax ${ }^{\circledR}$ da base de dados Micromedex da Truven Health Analyt$i c s^{12}$. As interações foram classificadas em relação à gravidade, documentação e tempo de início, adotando as especificações do Drug-Reax®:

- Gravidade - contra indicada (quando os medicamentos são contra indicados para uso concomitante); grave (quando a interação pode trazer riscos à vida do paciente e requer intervenção médica imediata); moderada (quando a interação pode resultar em exacerbação da condição clínica do paciente ou demandar alteração da terapia); leve (quando a interação pode ter efeitos clínicos limitados, sem demandar alterações na terapia medicamentosa); e desconhecida (quando não tem definição de grau de gravidade) ${ }^{12}$.

- Documentação científica - excelente (quando estudos controlados estabeleceram a existência da interação medicamentosa); boa (quando documentação forte sugere a existência da interação, mas faltam estudos bem controlados); regular (quando documentação disponível é escassa, mas as bases farmacológicas permitem suspeitar da interação); pobre (quando a documentação está limitada a estudos de casos); improvável (quando a documentação é pobre e faltam bases farmacológicas); e desconhecida (quando a documentação sobre a interação não é conhecida) ${ }^{12}$.

- Tempo de início - imediatas (quando o efeito da interação manifesta imediatamente ou nas primeiras 24 horas da administração conjunta dos medicamentos); e tardias (quando o efeito da interação manifesta 24 horas após a administração conjunta dos medicamentos) $)^{12}$.

Os fármacos envolvidos nas interações medicamentosas foram analisados em relação à adequação para idosos, segundo o Critério de Beers ${ }^{13}$, por ser o mais divulgado na literatura ${ }^{14}$ e por relacionar os medicamentos que devem ser evitados por idosos por apresentarem alto risco ${ }^{13}$.

O banco de dados foi estruturado na planilha do Excel (versão 2007). Foram realizadas dupla digitação e a validação dos dados para a identificação de possíveis erros. A análise estatística foi realizada empregando o software Statistical Analysis Software (SAS) Version 9.0, compreendendo análise estatística descritiva e teste de correlação de Pearson. Valores de $\mathrm{p} \leq 0,05$ foram considerados significativos.

O projeto de pesquisa foi autorizado pela Secretaria Municipal de Saúde de Ribeirão Preto e aprovado pelo Comitê de Ética em Pesquisa da Escola de Enfermagem de Ribeirão Preto da Universidade de São Paulo, sob o número de protocolo 00712212.6.0000.5393. Foi solicitado aos participantes da pesquisa a sua autorização mediante a assinatura do Termo de Consentimento Livre e Esclarecido.

\section{Resultados e Discussão}

A população do estudo foi constituída por 40 idosos, sendo $26(65,0 \%)$ pessoas do sexo feminino e $14(35,0 \%)$ do sexo masculino. A mediana da idade foi de 71,0 anos $(\mathrm{DP}=5,9)$. Com relação à escolaridade, $9(22,5 \%)$ eram analfabetos, $22(55,0 \%)$ responderam ter estudado de 1 a 4 anos, 8(20,0\%) estudaram de 5 a 11 anos e apenas $1(2,5 \%)$ tinha ensino superior.

O tempo médio do diagnóstico de hipertensão foi de 17,9 anos ( $\mathrm{DP}=9,6)$ e do uso de medicamentos foi de 18,3 anos $(\mathrm{DP}=8,9)$, sendo que $18(45 \%)$ usavam há mais de 20 anos. A média do número de diagnósticos foi de 2,9 (DP=1,1). Além de hipertensão arterial, 17 (42,5\%) dos pacientes apresentavam dislipidemia e Diabetes Mellitus. A média da pressão arterial sistólica foi de $150,9 \mathrm{mmHg}$ e da pressão diastólica 77,8 $\mathrm{mmHg}$.

Com relação ao número de medicamentos prescritos por paciente, a média foi de 7,5 (DP=2,8), totalizando 299 medicamentos para os 40 pacientes do estudo. Entre os medicamentos mais utilizados, o ácido acetilsalicílico (AAS) esteve presente em 31 $(77,5 \%)$ das prescrições médicas, seguido pela hidroclorotiazida - 25(62,5\%), sinvastatina - 24(60,0\%), omeprazol - 24(60,0\%), metformina - 22(55,0\%), enalapril - 22(55,0\%), glibenclamida - 11(27,5\%), captopril - 7(17,5\%), dentre outros.

$\mathrm{Na}$ análise da correlação de Pearson, o número de medicamentos apresentou correlação estatisticamente significante $(\mathrm{p}<0,05)$ com a presença de comorbidades $(r=0,314 ; p=0.0483)$, e com as interações medicamentosas $(\mathrm{r}=0.711 ; \mathrm{p}=0,001)$, ou seja, uma relação diretamente proporcional entre o número de medicamentos utilizados pelos pacientes e comorbidades e o número de interações medicamentosas. Ver Figura 1. 
Coeficiente de Correlação de Pearson $(r=0.31430 p=0.0483$

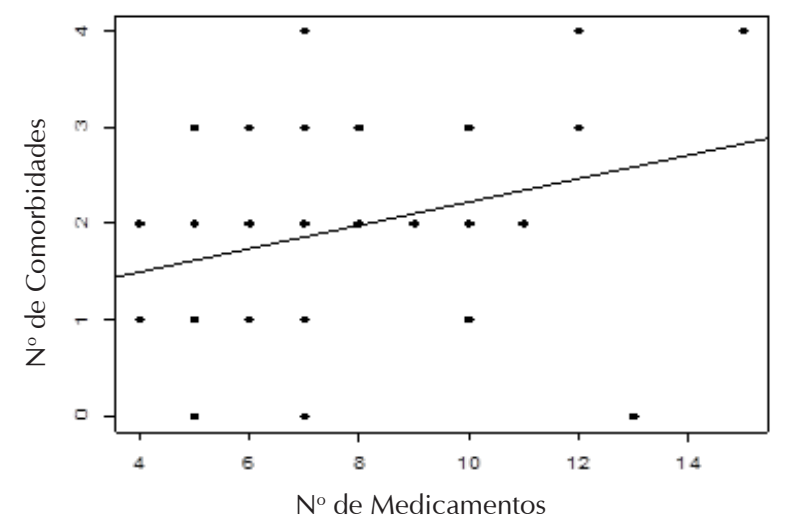

Coeficiente de Correlação de Pearson $(r=0.71104$ p<0.0001)

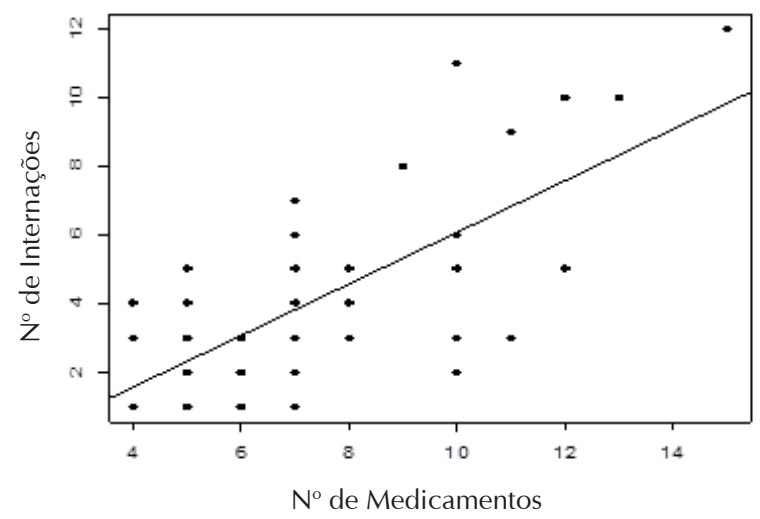

FIGURA 1: Gráficos de dispersão - número de comorbidades e número de interações medicamentosas versus número de medicamentos.

Para as interações medicamentosas obteve-se uma média de 4,2 $(\mathrm{DP}=2,9)$ por paciente. Todos os idosos incluídos no estudo apresentaram pelo menos uma interação e foram identificadas 169 interações medicamentosas. Segundo a gravidade, $139(82,2 \%)$ foram classificadas como moderadas, $29(17,2 \%)$ graves e 1(0,6\%) leve, totalizando 69 tipos diferentes. Quanto ao tempo de início, $54,7 \%$ das reações foram tardias e $22,7 \%$ foram imediatas. Em relação à documentação científica, $60,0 \%$ eram boas e $14,7 \%$ excelentes.

A interação grave mais frequente neste estudo foi a associação dos medicamentos anlodipino e sinvastatina, observada em 6(15,0\%) das prescrições médicas. As interações graves mais prevalentes no estudo e suas implicações clínicas, segundo o DrugReax ${ }^{\circledR}$, são apresentados na Tabela 1.
A interação moderada mais prevalente foi entre AAS e enalapril 17(42,5\%). As principais interações medicamentosas moderadas identificadas nas prescrições médicas e suas implicações clínicas, segundo o Drug-Reax ${ }^{\circledR}$, são mostradas na Tabela 2.

Entre os medicamentos utilizados pelos idosos, 37 fármacos participaram dos 69 tipos de interações medicamentosas identificadas no estudo. Os fármacos responsáveis pela maioria das interações foram o AAS (16 tipos); enalapril (8); hidroclorotiazida (9); glibenclamida (6); metformina (7); sinvastatina (5); anlodipino (4); captopril (4) e furosemida (4).

Com relação ao número de interações, o AAS somou 64; o enalapril 51; a hidroclorotiazida 41; a glibenclamida 26; a metformina 19; a sinvastatina 11; o anlodipino 9; o captopril 10 e a furosemida 11. Os

TABELA 1: Frequência absoluta e significância clínica das interações medicamentosas graves, identificas em prescrições para idosos. Ribeirão Preto, SP, 2012.

\begin{tabular}{lll}
\hline \multicolumn{1}{c}{ Interação medicamentosa } & f & \multicolumn{1}{c}{ Significância clínica } \\
\hline Anlodipino e Sinvastatina & 6 & Risco de miopatia e rabdomiólise \\
Ácido Acetilsalicílico e Fluoxetina & 3 & Aumento do risco de sangramento \\
Ácido Acetilsalićlico e Sertralina & 2 & Aumento do risco de sangramento \\
Alopurinol e Captopril & 2 & Reação de hipersensibilidade $\left.{ }^{*}\right)$ \\
Alopurinol e Enalapril & 2 & Reação de hipersensibilidade $\left.{ }^{*}\right)$ \\
Espironolactona e Enalapril & 2 & Risco de hipercalemia \\
Hidroclorotiazida e Digoxina & 2 & Risco de intoxicação digitálica \\
Ácido Acetilsalicílico e Varfarina & 1 & Aumento do risco de sangramento \\
Amiodarona e Sinvastatina & 1 & Aumento do risco de miopatia e rabdomiólise \\
Clonidina e Diltiazem & 1 & Aumento da incidência de bradicardia sinusal \\
Clopidogrel e Ácido Acetilsalicílico & 1 & Aumento do risco de sangramento \\
Espironolactona e Digoxina & 1 & Risco de intoxicação digitálica \\
Imipramina e Difosfato de Cloroquina & 1 & Aumento do risco de elevação do intervalo QT \\
Imipramina e Sertralina & 1 & Possibilidade de síndrome serotoninérgica \\
Metformina e Cimetidina & 1 & Risco de hipoglicemia \\
Propranolol e Clonidina & 1 & Aumento do risco bradicardia sinusal \\
Sinvastatina e Diltiazem & 1 & Aumento do risco de miopatia e rabdomiólise \\
\hline
\end{tabular}

(*)Farmacodermia, Stevens-Johnson 
TABELA 2: Frequência absoluta e significância clínica das principais interações medicamentosas moderadas, identificadas em prescrições para idosos. Ribeirão Preto, SP, 2012.

\begin{tabular}{lcll}
\hline \multicolumn{1}{c}{ Interação medicamentosa } & f & \multicolumn{1}{c}{ Significância clínica } \\
\hline Ácido Acetilsalićlico e Enalapril & 17 & Diminui a eficácia do enalapril \\
Ácido Acetilsalicílico e Glibenclamida & 11 & Excessiva hipoglicemia \\
Ácido Acetilsalicílico e Hidroclorotiazida & 11 & $\begin{array}{l}\text { Diminui a eficácia do diurético e do anti-hipertensivo; } \\
\text { aumenta o risco de toxicidade renal }\end{array}$ \\
Metformina e Enalapril & 11 & Acidose láctica hipercalêmica \\
Hidroclorotiazida e Enalapril & 10 & Hipotensão postural \\
Glibenclamida e Enalapril & 7 & Excessiva hipoglicemia \\
Ácido Acetilsalicílico e Furosemida & 5 & Diminui eficácia do diurético e do anti-hipertensivo \\
Glibenclamida e Hidroclorotiazida & 5 & Diminui a eficácia da glibenclamida \\
Hidroclorotiazida e Captopril & 4 & Hipotensão postural \\
Hidroclorotiazida e Diclofenaco & 3 & Reduz efeito anti-hipertensivo \\
\hline
\end{tabular}

medicamentos presentes nas interações, considerados inapropriados para idosos, segundo Critérios de Beers ${ }^{13}$, foram: glibenclamida (26), diclofenaco (9), fluoxetina (5), espironolactona ( $>25 \mathrm{mg} / \mathrm{dia})(5)$, digoxina $(>0,125 \mathrm{mg} / \mathrm{dia})(6)$, imipramina (2), amiodarona (1), doxazosina (1), fenobarbital (1) e metildopa (1).

O estudo demonstrou que o número de interações medicamentosas é diretamente proporcional ao número de medicamentos e comorbidades e evidenciou que a interação é um evento altamente frequente nos idosos atendidos, na atenção primária incluídos no estudo. Esse grupo etário, habitualmente, apresenta várias doenças crônicas que levam ao fenômeno da polipatologia e, consequentemente, a uma alta taxa de utilização de serviços de saúde e o emprego de múltiplos medicamentos. O medicamento contribui para a melhoria da qualidade de vida do idoso, mas pode, também, causar eventos adversos que levam a danos, alguns deles determinados por interações medicamentosas. $O$ consumo de múltiplos medicamentos contribui para a ocorrência de interações medicamentosas não desejáveis ${ }^{7,15-17}$.

Em uma abordagem quantitativa, o uso de múltiplos medicamentos está associado com interações medicamentosas em diferentes estudos ${ }^{15,17}$. A população investigada utilizou uma média de 7,5 medicamentos, o que permite classificar como uma polifarmácia alta. Em um estudo realizado na cidade de Blumenau, também no contexto da atenção primária, a média de medicamentos utilizados pelos idosos foi de 6,6 por idoso ${ }^{18}$.

Várias classificações são empregadas para classificar a polifarmácia, abrangendo polifarmácia menor - uso de 2 a 4 medicamentos e polifarmácia maior - uso de 5 ou mais medicamentos, ou ainda subdividida em polifarmácia baixa -2 a 3 medicamentos, moderada 4 a 5 medicamentos e alta - maior que 5 e subdividida em estratos ${ }^{1-3,4-5,6-8}$ e maior do que 9 medicamentos. $O$ termo polifarmácia também tem sido empregado com diversas conotações, tais como uso de medicamentos inapropriados, ocorrência de interação medicamentosa e efeito adverso de um fármaco tratado com outro fármaco ${ }^{10,11}$.
Os idosos apresentaram em média 2,9 diagnósticos referidos e, assim como em outros estudos, as doenças cardiovasculares foram aquelas que mais incidiram. Muitas dessas enfermidades, por serem crônicas, demandam alto custo na assistência à saúde, além de propiciarem o surgimento de complicações com grande interferência no grau de dependência e qualidade de vida das pessoas ${ }^{19}$.

O AAS foi o medicamento mais utilizado neste estudo, seguido pela hidroclorotiazida e sinvastatina. Assim, como em investigação realizada com idosos hipertensos no sul do Brasil, o AAS, o enalapril e a hidroclorotiazida estavam entre os medicamentos mais utilizados e responderam pelas principais interações medicamentosas de gravidade moderada ${ }^{18}$. Corroborando esse achado, uma revisão de literatura apontou que o AAS esteve entre os principais medicamentos com potencial de interação medicamentosa ${ }^{20}$.

As interações medicamentosas com AAS frequentemente estão superestimadas na maioria dos estudos, pois a dose utilizada pelos pacientes não é considerada nos estudos e pelos softwares empregados para identificação de interações. A dose é um fator importante nas interações com esse fármaco, porque muitas vezes ocorre somente em doses para uso como analgésico. Em idosos, o uso elevado é associado com a indicação como antiagregante plaquetário, cuja dose é geralmente de $100 \mathrm{mg}$. O AAS tem sido utilizado na prevenção de eventos tromboembólicos há muito tempo ${ }^{21}$, porém a dose que pode variar de 50 a $325 \mathrm{mg} / \mathrm{dia}^{22,23}$ continua sendo alvo de pesquisas e discussões até o presente momento, devido ao risco de efeitos adversos em doses maiores ou perda da eficácia terapêutica em doses baixas ${ }^{21}$.

As interações de AAS são dose-dependentes com os seguintes fármacos: enalapril, hidroclorotiazida, furosemida, captopril, espironolactona, diltiazem, verapamil e indapamida ${ }^{12}$. Essas interações ocorrem em doses analgésicas com significância clínica, pois compromete a resposta terapêutica do fármaco com a ação cardiovascular. Nesse sentido é importante alertar o idoso para os riscos da automedicação com AAS. 
As interações da glibenclamida com AAS e enalapril, detectadas neste estudo, têm potencial de causar hipoglicemia excessiva, demonstrando a relevância dessa interação e a necessidade de monitorar esses pacientes ou, quando disponível, substituir a sulfoniluréia por outra mais adequada a idosos ${ }^{12}$. A glibenclamida é um medicamento inapropriado para idosos, segundo o Critério de Beers, porque apresenta alto risco de produzir hipoglicemia grave prolongada em idosos ${ }^{13}$.

As interações medicamentosas graves, detectadas na presente investigação, podem comprometer a segurança da farmacoterapia do paciente, pois apresentam potencial de aumentar o risco de sangramento, cardiotoxicidade (bradicardia, intoxicação digitálica, alteração de intervalo QT) e reações de hipersensibilidade, submetendo o idoso a chance de apresentar reações adversas que podem comprometer sua funcionalidade e qualidade de vida ${ }^{12}$.

Medicamentos inadequados para idosos como fluoxetina, imipramina, clonidina, digoxina e amiodarona participam de interações graves, demonstrando a necessidade de reavaliar a indicação, analisar a dose empregada (manter digoxina $<0,125 \mathrm{mg}$ ), considerar alternativa terapêutica e identificar estratégias de monitorização quando o fármaco não apresentar alternativa, como frequentemente ocorre com o uso da amiodarona ${ }^{12}$. Essas estratégias de monitorização se aplicam a todas as interações medicamentosas.

Em virtudes das alterações da senescência e da senilidade, o idoso apresenta alterações fisiológicas, destacando as relativas à função renal, que podem comprometer a farmacocinética e farmacodinâmica dos medicamentos. As interações de losartana + diclofenaco e captopril + diclofenaco, detectadas neste estudo, além de comprometer o efeito anti-hipertensivo, apresenta potencial de induzir disfunção renal no idoso. Além disso, o diclofenaco é um fármaco inadequado para idoso, pois também aumenta o risco de sangramento gastrintestinal ${ }^{12}$. Os critérios de Beers ${ }^{13}$ destacam que idosos maiores de 75 anos que utilizam corticosteróides, antiagregantes e anticoagulantes concomitantes com anti-inflamatórios não esteroidais apresentam riscos de sangramento gastrintestinal e ressaltam que o risco aumenta com o uso prolongado.

Anlodipino e sinvastatina e amiodarona + sinvastatina, quando administrados conjuntamente, apresentam interação grave o que pode acarretar em aumento da exposição à sinvastatina e aumento do risco de miopatias, inclusive rabdomiólise. Se for necessário o uso desses medicamentos, concomitantes, a dose de sinvastatina não deve exceder $20 \mathrm{mg} / \mathrm{dia}$, conforme consta de alerta publicado pelo Food and Drug Administration (FDA) ${ }^{12,24}$.

Na prática assistencial, muitas vezes, a interação medicamentosa é determinada pela condição clínica dos pacientes ${ }^{25,26}$. No caso do idoso, pela complexidade da farmacoterapia e múltiplas patologias, é geralmente indicado o uso concomitante de fármacos, mas para garantir uma farmacoterapia segura e efetiva é importante que a equipe de saúde saiba identificar as interações e reconhecer as estratégias de monitorização.

Vale destacar que o cuidado é elemento estrutural na prática de enfermagem, portanto torna-se necessário identificar, estudar, conhecer e analisar as práticas de cuidados que são realizadas pelos enfermeiros no campo da atenção primária em saúde ${ }^{27}$. O enfermeiro pode atuar e contribuir para melhorar a qualidade dos serviços prestados nessa área.

Entre as limitações dessa investigação, destacase o delineamento e o método empregado para análise das interações, que devem ser considerados na interpretação dos dados. A identificação de interações medicamentosas, empregando software, detecta apenas as interações potenciais, o que não significa que os possíveis eventos adversos manifestem-se clinicamente em todos os pacientes com uma interação fármaco-fármaco potencial. Os softwares são importantes instrumentos para verificar interações medicamentosas potenciais, mas geralmente produzem alto nível de sinal que podem indicar maior prevalência de interações ${ }^{25}$. Entretanto, um aspecto diferencial dessa investigação que contribui para a validade dos resultados obtidos, é ter empregado um software que apresenta sensibilidade e especificidade adequada para identificação de interações medicamentosas potenciais ${ }^{25,26}$.

A identificação das interações foi realizada sem observar a dose de medicamento e o tempo de tratamento. Sendo assim, é possível que a prevalência tenha sido superestimada, porque algumas interações podem ser doses dependentes e os processos de inibição e indução enzimática são tempo dependentes. Esses processos enzimáticos são determinantes nas interações farmacocinéticas, envolvendo o metabolismo de fármacos. Outra limitação foi a amostra de conveniência, abrangendo apenas uma UBS, aspecto que restringe a generalização dos resultados.

Sendo assim, novos estudos podem ser realizados abrangendo uma população maior, observando-se a dose do medicamento e o tempo de tratamento, a fim de ampliar o conhecimento dos profissionais de saúde, garantindo a segurança do paciente.

\section{Conclusão}

O número de interações medicamentosas nas prescrições dos pacientes deste estudo foi diretamente proporcional ao número de medicamentos e de comorbidades. A ocorrência de interações fármaco-fármaco foi elevada nos pacientes investigados, abrangendo interações de significância clínica. As interações detectadas podem apresentar e induzir eventos adversos como: hipoglicemia, cardiotoxicidade, inefetividade 
da terapia anti-hipertensiva e sangramentos que comprometem a segurança da farmacoterapia do idoso, interferindo significativamente na funcionalidade e na qualidade de vida relacionada à saúde.

Dessa forma, o estudo contribuiu para conhecer o perfil das interações medicamentosas em idosos hipertensos, tornando-se ferramenta importante para o planejamento de ações para melhoria da segurança do paciente hipertenso crônico em uso de múltiplos medicamentos em tratamento na atenção primária.

\section{REFERÊNCIAS}

1.Instituto Brasileiro de Geografia e Estatística. Censo Demográfico 2010: Características gerais da população, religião e pessoas com deficiência [Internet]. Rio de Janeiro; 2010 [citado em 15 set 2014]. Disponível em: $\mathrm{ftp}: / / \mathrm{ftp}$.ibge.gov.br/Censos/Censo_Demografico_2010/ Caracteristicas_Gerais_Religiao_Deficiencia/caracteristicas_religiao_deficiencia.pdf.

2.Nóbrega OT, Karnikowski MGO. A terapia medicamentosa no idoso: cuidados na medicação. Cien Saude Colet [Internet]. 2005 [citado em 05 set 2014]; 10: 309-13. Disponível em: http://www.scielo.br/scielo.php?script=sci_ arttext\&pid=S1413-81232005000200008\&lng=en. 3.Sokol MC, McGuigan KA, Verbrugge RR, Epstein RS. Impact of medication adherence on hospitalization risk and healthcare cost. Med Care. 2005; 43: 521-30.

4.Costa SC, Pedroso ERP. A prescrição de medicamentos para idosos internados em serviço de clínica médica: atualização. Rev Med Minas Gerais. 2011; 21: 201-14. 5.Santos TRA, Lima DM, Nakatani AYK, Pereira LV, Leal GS, Amaral RG. Consumo de medicamentos por idosos, Goiânia, Brasil. Rev Saude Publica. 2013, 47: 94-103. 6.Sociedade Brasileira de Hipertensão. Diretrizes Brasileiras de Hipertensão Arterial VI. Rev Hipertensão [Internet]. 2010 [citado em 05 abr 2014]. Disponível em: http://www.sbh.org.br/pdf/diretrizes_final.pdf. 7.Secoli SR. Polifarmácia: interações e reações adversas no uso de medicamentos por idosos. Rev Bras Enferm. 2010; 63: 136-40.

8.Amaral DMD, Perassolo MS. Possíveis interações medicamentosas entre os antihipertensivos e antidiabéticos em participantes do Grupo HIPERDIA de Parobé, RS (Uma análise teórica). Rev Ciênc Farm Básica Apl. 2012; 33: 99-105.

9.Carreira CFS, Barrêto VFT, Moura APG, Silva PRJ, Teixeira NAM, Canavieiras AS. Interações medicamentosas: um relato de caso sobre a avaliação e intervenção farmacêutica. In: 11. Encontro de Iniciação à Docência; 2008; João Pessoa (PB): UFPB, 2008.

10.Salazar JA, Poon I, Nair M. Clinical consequences of polypharmacy in elderly: expect the unexpected, think the unthinkable. Expert Opinion Drug Safety. 2007; 6: 695-704. 11.Silva AL. Estudo de utilização de medicamentos por idosos brasileiros [dissertação de mestrado]. Belo Horizonte (MG): Faculdade de Farmácia da Universidade Federal de Minas Gerais; 2009.

12.Micromedex Healthcare Series [Internet]. Truven
Health Analytics. 2012 [citado em 05 set 2014]. Available in: http://www-micromedexsolutions-com.ez67. periodicos.capes.gov.br/micromedex2/librarian/.

13.American Geriatrics Society 2012 Beers Criteria Update Expert Panel. American Geriatrics Society updated Beers Criteria for potentially inappropriate medication use in older adults. J Am Geriatr Soc. 2012; 60: 616-31. 14.Soares MA, Fernandez-Llimos F, Cabrita J , Morais J. Tools to evaluate potentially inappropriate prescription in the elderly: a systematic review. Acta Med Port. 2011; 24: 775-84. 15.Obreli Neto PR, Nobili A, de Lyra DPJ, Pilger D, Guidoni CM, Oliveira Baldoni A, et al. Incidence and predictors of adverse drug reactions caused by drug-drug interactions in elderly outpatients: a prospective cohort study. J Pharm Pharmaceut Sci. 2012; 15: 332-43.

16.Passarelli MCG, Jacob Filho W. Reações adversas a medicamentos em idosos: como prevê-las? Einstein. 2007; 5: 246-51.

17.Lea M, Rognan SE, Koristovic R, Wyller TB, Molden E. Severity and Management of Drug-Drug Interactions in Acute Geriatric Patients. Drugs Aging. 2013; 30:721-7. 18.Codagnone Neto V, Garcia VP, Santa Helena ET. Possible pharmacological interactions in hypertensive and/ or diabetic elderly in family health units at Blumenau (SC). Braz J Pharm Sci. 2010; 46: 795-804.

19.Marin MJS, Cecílio LCO, Perez AEWUF, Santella F, Silva CBA, Filho Gonçalves JR, et al. Caracterização do uso de medicamentos entre idosos de uma unidade do Programa Saúde da Família. Cad Saúde Pública. 2008; 24: 1545-55. 20.Silva LD, Santos MM. Interações medicamentosas em unidade de terapia intensiva: uma revisão que fundamenta o cuidado do enfermeiro. Rev enferm UERJ. 2011; 19: 134-9.

21.Araujo BG, Menezes AC. Dose do AAS como Antiagregante plaquetário. In: Souza PM, Araujo BG, Silva LP, organizadores. Farmacologia clínica: textos informativos. Brasília (DF): Universidade de Brasilia; 2012. p. 88-90.

22.Ansara AJ, Nisly SA, Arif SA, Koehler JM, Nordmeyer ST. Aspirin dosing for the prevention and treatment of ischemic stroke: an indication-specific review of the literature. Ann Pharmacother. 2010; 44: 851-62. 23.Ministério da Saúde (Br). Secretaria de Ciência, Tecnologia e Insumos Estratégicos, Departamento de Assistência Farmacêutica e Insumos Farmacêuticos. Formulário Terapêutico Nacional: Rename 2008. Brasília (DF): Editora MS; 2008.

24.Food Drug Administration. Drug safety communication: new restrictions, contraindications, and dose limitations for Zocor (simvastatin) to reduce the risk of muscle injury, 2011. [cited 2014 Set 15]. Avaible : http://www. fda.gov/Drugs/DrugSafety/ucm256581.htm.

25.Reis AM, Cassiani SH. Evaluation of three brands of drug interaction software for use in intensive care units. Pharm World Sci. 2010; 32: 822-8.

26.Vonbach P, Dubied A, Krähenbühl S, Beer JH. Evaluation of frequently used drug interaction screening programs. Pharm World Sci. 2008; 30: 367-74.

27.Ferreira VA, Acioli S. O cuidado na prática do enfermeiro no campo da atenção primária em saúde: produção científica. Rev enferm UERJ. 2009; 17: 506-9. 